Check for updates

Cite this: RSC Adv., 2019, 9, 10386

\title{
Accelerated biodegradation of PLA/PHB-blended nonwovens by a microbial community $\dagger$
}

\author{
Yalan Liu, ${ }^{a}$ Zhicheng Zhan, ${ }^{\mathrm{b}}$ Haixian Ye, ${ }^{\mathrm{b}}$ Xiaoshan Lin, ${ }^{\mathrm{a}}$ Yurong Yan ${ }^{\star b}$ \\ and Yi Zhang $\mathbb{D}$ *a
}

In this study, the accelerated biodegradation of PLA/PHB (polylactic acid/polyhydroxybutyrate)-blended nonwovens was investigated in the presence of a microbial community. The PLA/PHB-blended nonwovens were buried in natural soil for 56 days, with soil samples collected for subsequent bacterial community domestication. The tensile strength and elongation at break of the PLA/PHB-blended nonwovens as well as the $\mathrm{CO}_{2}$ generated by the Gen III and natural soil communities were determined to assess the degradation rates of the polymer samples. After incubation for 15 days with the Gen III soil bacterial suspension, the surfaces and fibrous structure of nonwovens and the fibers within the nonwovens exhibited distinct changes. In addition, the amount of $\mathrm{EvCO}_{2}$ reached $566.79 \mathrm{mg}$, the tensile strength decreased from $10.95 \pm 0.7$ to $2.57 \pm 0.31 \mathrm{MPa}$, a loss of $77 \%$, and the elongation at break changed from $5.32 \pm 0.45$ to $7.07 \pm 1.04 \%$. The $16 \mathrm{~S}$ rRNA pyrosequencing results showed that Proteobacteria and Firmicutes were the 2 most important bacterial phyla in the Gen III community, accounting for 80.4 and $19.4 \%$ of the total classified sequences, respectively. The results of this study demonstrate that compared to a natural soil microbial community, the domesticated strains in the Gen III community, especially members of the phyla Proteobacteria and Firmicutes, are useful in accelerating the degradation of PLA/PHB-blended nonwovens.

Received 27th December 2018 Accepted 28th March 2019

DOI: 10.1039/c8ra10591j

rsc.li/rsc-advances pollution in the oceans, with Asia accounting for $80 \%$ of the total volume. ${ }^{3}$ The amount of marine plastic pollution derived from land-based sources is from 4.8 to 12.7 million metric tons annually. ${ }^{4}$ Thus, the demand for biodegradable plastics has increased in recent years, with an increased awareness of the plastic waste problem and its impact on the environment eliciting new interest in the area of degradable polymers. ${ }^{5}$

Poly(L-lactic acid) (PLA) is a bio-based polymer that has attracted a great deal of attention in recent years due to its many useful properties, including its high transparency, high tensile strength and excellent biodegradability, with its final degradation products consisting of carbon dioxide, water and other small molecules under composting conditions. ${ }^{6}$ However, because of a number of drawbacks of PLA, such as its low impact strength and poor heat resistance, it needs to be modified in some circumstances. A number of polymers are used to increase the toughness of PLA, such as poly(butylene adipate-co-terephthalate) (PBAT), ${ }^{7}$ PCL, polyurethane, polyamide elastomer (PAE) ${ }^{8}$ starch, ${ }^{9}$ GMA-based copolymers, thermoplastic elastomers and GMA grafted copolymers. ${ }^{\mathbf{1 0}}$

To improve the toughness and alter the biodegradation period, the use of biodegradable polyhydroxybutyrate (PHB) was evaluated in a previous study. ${ }^{11}$ In 1926, the French scientist Lemoigne first discovered and synthesized the most common monomer in Bacillus megaterium, polyhydroxybutyrate (PHB), the first PHA identified and studied. Some properties of PHB are 
the same as those of the conventional plastic polypropylene, such as tensile strength, impact strength, degree of crystallinity, and melting temperature, although PHB is more brittle than PP and is more susceptible to thermal degradation. Because of its biodegradability, biocompatibility and nontoxicity, PHB is a promising candidate in biomedical and agricultural applications. $^{\mathbf{1 2}}$

Objective evaluations of the biodegradability of biodegradable materials is an effective means of guiding the rational application of materials, with degradation in natural and specific accelerated degradation environments being commonly investigated. In natural environments, a long period of time is required, sometimes even years, whereas the use of specific environments to accelerate degradation by bacteria that break down organic matter allows key aspects of the degradation process to be investigated and evaluated. In 1977, microbial enzymes were identified that can attack synthetic, manmade polymers. ${ }^{\mathbf{1 3}}$ However, the activity of these enzymes appeared to be limited to the biodegradation of aliphatic polyesters, which have few useful properties, excluding the use of biodegradable plastics from many applications. In 1995, Witt and Müller discovered aliphatic-aromatic copolyesters with a specific composition could be degraded by microorganisms, and they concluded that great improvements could be made in the characteristics of polymers. ${ }^{\mathbf{1 4}}$ The biodegradation behavior of PLA and its blends has been investigated in a number of studies. Weng et al. studied the biodegradation of PLAs with different chemical structures under controlled composting conditions, ${ }^{15}$ while Rudnik and Briassoulis ${ }^{16}$ compared the biodegradation of PLA and PHA in soil environments and under composting conditions. Their results showed that these polymers could be biodegraded at a high rate in both real soil environments and under composting conditions. Thus, the microorganisms in soil and compost may play a major role in the biodegradation of renewable polymers.

In contrast, the degradation of PLA/PHB-blended nonwovens under natural conditions, including in soil environments and under composting conditions, was observed to occur at a very low rate, ${ }^{17}$ which is a major drawback limiting their usefulness. However, accelerating the biodegradation process would overcome this limitation and make these blends much more attractive. As the PLA/PHB $=80 / 20$ is the most commonly used ratio in manufacturing applications, we applied the PLA/PHBblended nonwovens with the same ratio in this study. In this study, we domesticated a soil bacterial community to enhance the efficiency and shorten the duration of the biodegradation process of PLA/PHB-blended nonwovens, the preliminary results of which showed that their biodegradation occurred much faster and more completely in domesticated bacterial suspensions than in a natural environment. These results indicated that the domesticated bacterial community was a strong driver promoting this biodegradation process.

Identifying the microbial communities involved in enhancing the biodegradation process and studying the microbial community composition changes during this process are necessary to reveal the mechanism promoting the rapid biodegradation of polymers. The results of previous studies demonstrated that advanced molecular techniques are provided powerful tools for detecting bacterial community structure and diversity in environmental samples, as well as for evaluating the biodegradation of PLA/PHB-blended nonwovens. ${ }^{18}$ In this study, high-throughput pyrosequencing was performed to quantify the abundance, diversity and structure of a domesticated bacterial community that promoted the rapid biodegradation of polymers from renewable biomass.

\section{Experimental}

\section{Materials}

The LB culture medium used in this study was produced by Guangdong Huankai Microbial Co., Ltd., while the chemicals (standard Q/HK3) $\quad \mathrm{K}_{2} \mathrm{HPO}_{4} \cdot 3 \mathrm{H}_{2} \mathrm{O}$ (Guangzhou Chemical Reagent Factory), $\mathrm{MgSO}_{4} \cdot 7 \mathrm{H}_{2} \mathrm{O}$ (Guangzhou Chemical Reagent Factory), $\mathrm{MnSO}_{4} \cdot \mathrm{H}_{2} \mathrm{O}$ (Shantou Guanghua Chemical Reagent Factory), $\mathrm{NH}_{4} \mathrm{NO}_{3}$ (Guangzhou Chemical Reagent Factory), and $\mathrm{NaCl}$ (Guangzhou Chemical Reagent Factory) were all analytical reagents and used without additional purification. Chloroform was purchased from Shantou Xilong Chemical Co. Ltd. The PLA (grade 6202D) and PHB (grade 18J45) used in this study were produced by Natureworks LLC (USA) and BASF SE (Germany), respectively.

\section{PLA/PHB-blended nonwovens}

The PLA/PHB-blended nonwoven material was prepared on a traditional spunbonded nonwoven production line with a PLA/PHB weight ratio of $80 / 20$ and was kindly provided by U.S. Pacific, China. The nonwoven samples had a basic weight of $40 \mathrm{~g} \mathrm{~m}^{-2}$ and were used as received other than being cut into $30 \mathrm{~cm} \times 20 \mathrm{~cm}$ specimens for biodegradation assays.

\section{Biodegradation of PLA/PHB buried in a soil environment}

The soil study site was located in Guangzhou University Town, China, at a longitude of 113.4 and a latitude of 23.0 according to GB/T 18 006. Nonwoven specimens were buried in ordinary orchard soil at a depth of 15-20 cm for 56 days, with 3 sample pieces excavated for testing every 14 days. After being washed with distilled water 3 times, the samples were dried at room temperature and then stored in a refrigerator for further testing. ${ }^{19}$ The average temperature and relativity humidity during the testing period are presented in Fig. S2.† During the experiment period (from March to May), the temperature in the Panyu District of Guangzhou City ranged from 16.6 to $30.5^{\circ} \mathrm{C}$, with an average temperature was $23.89{ }^{\circ} \mathrm{C}$, while the humidity ranged from 51.5 to $96 \%$, with an average humidity of $80.79 \%$ (Fig. S2†).

\section{Soil samples collection and bacterial community domestication}

Soil samples were collected from an orchard at the same longitude and latitude at a depth of $15-20 \mathrm{~cm}$ and were sieved through a $2 \mathrm{~mm}$-sized sieve to remove particles larger than 2 mm. ${ }^{20}$ The soil was prepared according to ISO-11274 1998, and the $\mathrm{pH}$ of the suspensions was tested according to ISO 10390 
2005. LB culture medium was added to the suspensions to form uniform suspensions and then was sterilized at $115{ }^{\circ} \mathrm{C}$ for $20 \mathrm{~min}$.

Sterile LB culture medium (2.1 g) and $100 \mathrm{~mL}$ of distilled water was added to a $500 \mathrm{~mL}$ flask, sterilized at $115{ }^{\circ} \mathrm{C}$ for $20 \mathrm{~min}$ and then cooled down for further use. Next, $10 \mathrm{~g}$ of screened soil samples were added into the sterilized LB liquid medium and cultured for 24 hours with shaking at $190 \mathrm{rpm}$ at $37{ }^{\circ} \mathrm{C}$, after which the first generation of the soil bacterial suspension ('Gen I') was collected. The second generation of bacteria 'Gen II' was derived from a suspension culture of liquid medium seeded with $1 \mathrm{~mL}$ of 'Gen I' inoculated into $100 \mathrm{~mL}$ of sterilized LB liquid medium using the same conditions described above. The third and the fourth generations of bacteria ('Gen III' and 'Gen IV', respectively) were prepared in the same way and so on until the composition of the domesticated bacterial community stabilized (Fig. S1, Table S1†). The final resulting bacterial suspensions were stored at $4{ }^{\circ} \mathrm{C}$. Because the 'Gen III' community exhibited a stable composition, it was used as an inoculant in further tests.

\section{Biodegradation tests of polymer samples in the soil bacterial suspensions}

Jars with a $500 \mathrm{~mL}$ working volume were used as reactors. The jars were covered and sealed with 8 layers of gauze, sterilized for $20 \mathrm{~min}$ at $115{ }^{\circ} \mathrm{C}$, and then allowed to cool. Each reactor was filled with $7.35 \mathrm{~g}$ of LB culture medium, $350 \mathrm{~mL}$ of distilled water, and 5 pieces of nonwoven samples, which were all completely immersed into the liquid medium. One milliliter of the 'Gen III' suspensions was inoculated into the jar and was cultured for 24 hours with shaking at $150 \mathrm{rpm}$ at room temperature. All of the reactors were cultured at room temperature for 24 hours in a shaker and incubated at room temperature for 15 days. Samples were collected from the reactor every 3 days and were washed carefully with distilled water 4 times, dried at room temperature and then stored in a refrigerator for further testing. ${ }^{21}$

\section{Assessment of accelerated biodegradation}

Mechanical properties test. To evaluate the degradation rates of the polymer samples, the tensile strength and elongation at break of the PLA/PHB-blended nonwovens samples were determined using an INSTRON 5969 tester (USA) according to the GB/T3923.1 1997 method at $20 \pm 2{ }^{\circ} \mathrm{C}$ and 65\% humidity,

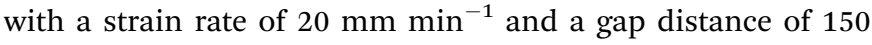
$\mathrm{mm} .{ }^{22}$ All of the tests were conducted in triplicate, and before testing, the samples were conditioned in the testing environment for 24 hours.

Morphological study. The morphology of the nonwoven specimens biodegraded for different lengths of time were determined using a S-3000N SEM (Hitachi, Japan) at $20 \mathrm{kV} .^{23}$ The surfaces of all the samples were coated with gold prior to SEM examination.

\section{Evaluation of the biodegradability of the nonwoven blends}

According to ISO 14852 (ref. 24), determination of the $\mathrm{CO}_{2}$ generated during aerobic polymer biodegradation is the most commonly used method to assess the degree of biodegradation. The detection device shown in Fig. 1 was designed according to ISO 14852 and consisted of an air pump, air $\mathrm{CO}_{2}$ absorption bottles, a bioreactor and evolved $\mathrm{CO}_{2}$ absorption bottles. The air was passed through the absorption bottles containing $\mathrm{NaOH}$ solution at a rate of $50-100 \mathrm{~mL} \mathrm{~min}{ }^{-1}$ to eliminate $\mathrm{CO}_{2}$, after which the $\mathrm{CO}_{2}$-free air entered the bioreactor. The degradation of the PLA/PHB-blended nonwovens was carried out using a thermostatic magnetic stirrer to control the temperature and rotation speed. Evolved $\mathrm{CO}_{2}$ from the degraded PLA/PHBblended nonwovens was absorbed by $\mathrm{Ba}(\mathrm{OH})_{2}$ in the absorption bottles, and the resulting $\mathrm{BaCO}_{3}$ was collected to quantity the evolved $\mathrm{CO}_{2}$.

Five grams of the PLA/PHB-blended samples was cut into pieces and placed into the bioreactors containing $200 \mathrm{~mL}$ of detection solution. Next, $1.0 \mathrm{~mL}$ of the mixed bacterial suspension was used to inoculate the bioreactor and was cultured at $30{ }^{\circ} \mathrm{C}$ for a specific length of time. Following the biodegradation experiments, the weight of the collected $\mathrm{BaCO}_{3}$ was quantified to calculate the amount of evolved $\mathrm{CO}_{2}\left(\mathrm{EvCO}_{2}\right)$ generated during the biodegradation assay. The theoretical evolved $\mathrm{CO}_{2}\left(\mathrm{ThCO}_{2}\right)$ can be calculated from the initial input amount of the blended nonwoven sample and the total organic carbon (TOC) of that material. A control, untreated sample was also run at the same time as the treatments.

\section{Total DNA extraction and PCR amplification}

Microbes in 'Gen III' were collected for DNA extraction, which was performed using a soil total DNA isolation kit (OMEGA BioTek, USA) following the manufacturer's instructions. The specific primers used to amplify the V3 region (161 bp) of the bacterial 16S rRNA gene were F341 (5'-CCTACGGGAGGCAGCAG-3') and R534 (5'-ATTACCGCGGCTGCTGG- $\left.3^{\prime}\right) .{ }^{25}$ The PCR amplification system included the following: $10 \times$ PCR buffer, $0.2 \mathrm{mmol} \mathrm{L}^{-1} \mathrm{dNTPs}$, upstream and downstream primers $(0.4$ $\mu \mathrm{mol} \mathrm{L}{ }^{-1}$ each), $1 \mathrm{U}$ Ex Taq polymerase (TaKaRa), $10 \mathrm{ng}$ of the sample DNA as template, and water to a total volume of $25 \mu \mathrm{L}^{26}$ The amplification steps were as follows: a $95{ }^{\circ} \mathrm{C}$ predenaturation for $5 \mathrm{~min}$ followed by 25 cycles of $94{ }^{\circ} \mathrm{C}$ for $45 \mathrm{~s}, 65-55^{\circ} \mathrm{C}$ for $30 \mathrm{~s}$ (the annealing temperature was progressively reduced from 65 to $55{ }^{\circ} \mathrm{C}$ at a rate of $1{ }^{\circ} \mathrm{C}$ every 2 cycles, with 5 cycles at $55^{\circ} \mathrm{C}$ ), and $72{ }^{\circ} \mathrm{C}$ for $1 \mathrm{~min}$, with a final extension at $72{ }^{\circ} \mathrm{C}$ for $8 \mathrm{~min}$.

\section{High-throughput pyrosequencing analysis}

The DNA samples were sent to Sangon Biotech Co. Ltd. (Shanghai, China) for pyrosequencing of the V3 region of the 16S rRNA gene following the 454 GS-FLX Titanium protocol (454 Life Sciences/Roche Diagnostics, CT, USA), which yields reads lengths of $\sim 400 \mathrm{bp}^{27,28}$ The paired sequences were combined using FLASH (http://ccb.jhu.edu/software/FLASH) with the minimum overlap value set to $10 \mathrm{bp}$ and the default settings used for the other parameters. The PCR artifacts were denoised 


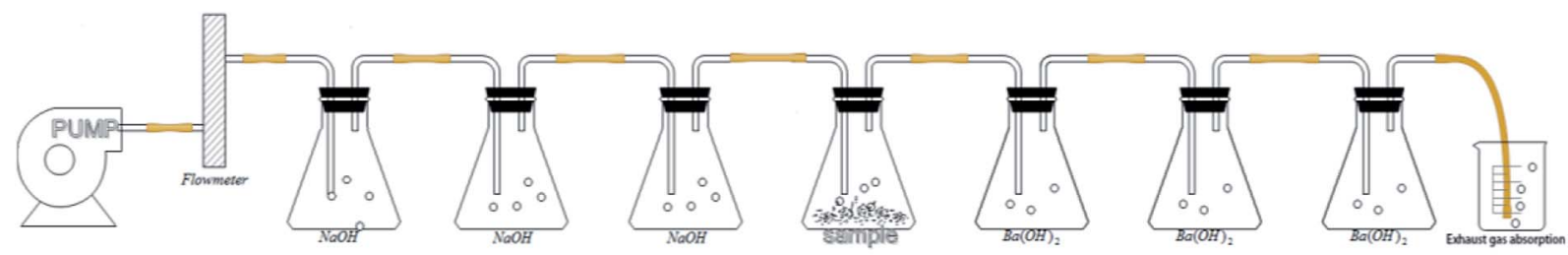

Fig. 1 Device used to detect the amount of $\mathrm{CO}_{2}$ evolved by PLA/PHB-blended nonwovens during the degradation process.

by eliminating the low-quality sequences using Trimmomatic. ${ }^{29}$ In UPARSE, the quality-filtered sequences were clustered into $d e$ novo OTUs at a $97 \%$ similarity threshold, with chimeric sequences removed using Uchime. ${ }^{30}$ USEARCH was used to map the reads to the OTUs to generate an OTU abundance table. We removed one outlier with a very low sequence count and microbial diversity. MOTHUR was used to construct rarefaction curves and to calculate ecological indices of the Chao1 estimator and Shannon diversity/richness. The sequences were classified using RDP classifier at a 80\% confidence threshold for sequences longer than $200 \mathrm{bp}$. The genera obtained from the RDP classifier were clustered via the complete linkage hierarchical clustering technique using the R package Hclust CLUST (http://sekhon.berkeley.edu/stats/html/hclust.htmL). The goal of the analysis was to distinguish between the bacterial species at genus, family, order, class or phylum level rather than to generate complete profiles of bacterial genes.

\section{Results and discussion}

\section{Changes in the mechanical properties and physical appearance of the PLA/PHB-blended nonwovens}

During degradation, the primary polymer chains break down or become crosslinked, which depends on the mechanism of the degradation, and all degradation processes impact the mechanical properties of products. The tensile strength and the elongation at break values of the tested PLA/PHB-blended nonwoven samples are reported in Table 1.

The results presented in Table 1 show that a decrease in tensile strength occurred under both degradation conditions, but opposing trends and fluctuations in the changes were observed for the elongation at breaks. When the samples were degraded in Gen III soil bacterial suspension, the tensile strength decreased from $10.95 \pm 0.77$ to $2.57 \pm 0.31 \mathrm{MPa}$, a loss of $77 \%$, while the elongation at break fluctuated but increased from $5.32 \pm 0.45$ to $7.07 \pm 1.04 \%$. For samples buried in the soil, slower decreasing trends were observed for both the tensile strength (from $10.95 \pm 0.77$ to $7.73 \pm 0.54 \mathrm{MPa}$, a loss of $30 \%$ ) and in the elongation at break values (from $5.32 \pm 0.45$ to $4.45 \pm$ $0.54 \%$ ). When the tested samples were immersed in liquid (Gen III), the bonding points in the nonwovens exhibited a debonding behavior that decreased the tensile strength of nonwovens and increased the sliding between fibers inside the nonwovens, which increased the elongation at break values of the tested samples. However, because the swelling and soaking behavior of liquid is not appreciable in soil, biodegradation is the only mechanism through which the fibers can be degraded. Thus, the observed breakage of nonwovens was only related to the biodegradation of the fibers, and the variation showed changes in both tensile strengths and elongation at break values.

Comparing with the degradation observed in Gen III soil bacterial suspension and in the buried soil environment, we observed that the tensile strength of the PLA/PHB-blended nonwovens in the buried soil environment for 45 days was the same as that of the samples incubated with the Gen III soil bacterial suspension for 3 days. The variation in the nonwoven samples incubated in the bacterial suspension compared to that observed in the samples buried in natural soil is further evidence that the degradation of the PLA/PHB-blended nonwovens can be accelerated in soil bacterial suspensions.

Table 1 The tensile strength and the elongation at break values of the tested PLA/PHB blended nonwovens treated under the indicated conditions

\begin{tabular}{|c|c|c|c|}
\hline Test type & Incubation time (days) & Tensile strength (MPa) & $\begin{array}{l}\text { Elongation at } \\
\text { break (\%) }\end{array}$ \\
\hline \multirow[t]{6}{*}{ Degradation in soil bacterial suspensions Gen III } & 0 & $10.95 \pm 0.77$ & $5.32 \pm 0.45$ \\
\hline & 3 & $6.82 \pm 0.44$ & $5.33 \pm 0.32$ \\
\hline & 6 & $5.69 \pm 0.70$ & $7.94 \pm 1.09$ \\
\hline & 9 & $4.96 \pm 0.54$ & $7.60 \pm 1.70$ \\
\hline & 12 & $2.83 \pm 1.00$ & $4.67 \pm 1.14$ \\
\hline & 15 & $2.57 \pm 0.31$ & $7.07 \pm 1.04$ \\
\hline \multirow[t]{5}{*}{ Degradation in the soil burial environment } & 0 & $10.95 \pm 0.77$ & $5.32 \pm 0.45$ \\
\hline & 14 & $9.40 \pm 0.89$ & $7.26 \pm 0.71$ \\
\hline & 28 & $8.48 \pm 1.41$ & $5.82 \pm 0.71$ \\
\hline & 42 & $8.50 \pm 1.00$ & $4.80 \pm 1.00$ \\
\hline & 56 & $7.73 \pm 0.54$ & $4.45 \pm 0.54$ \\
\hline
\end{tabular}




\section{Morphological analysis of the degradation of PLA/PHB-} blended nonwovens

The morphology of the PLA/PHB (80/20) blends showed a seaisland pattern with of island phase of PHB and sea phase of PLA. $^{31}$ The SEM image in Fig. 2 shows the morphology of the PLA/PHB-blended nonwovens. The morphologies of test samples that were buried in the buried soil environment for 56 days and those of samples that were exposed to the Gen III soil bacterial suspensions for 15 days in the laboratory are shown in Fig. 2A and B, respectively. Morphological changes can be clearly observed for all of the samples. At the start of biodegradation (0 day), obviously regular, homogenous fibers with smooth surfaces inside the PLA/PHB-blended nonwovens were observed. After 56 days of being buried in the soil, slight scratches appeared in the fiber surfaces, and the fibrous structure of the test samples had undergone minimal damage. These changes indicated that the biodegradation processes had begun, albeit slowly. However, after incubation for 15 days with the Gen III soil bacterial suspension, the surfaces and fibrous structure of the nonwovens and fibers within the nonwovens exhibited distinct changes. The scanning electron microscopy results showed that pores had appeared on the surface of the samples, and microcracks were also visible within the fibrous reticular structure. The presence of these phenomena indicated that the PLA/PHB-blended nonwovens had rapidly degraded in the domesticated bacterial suspension.

For the dispersion of PHB (island) in the PLA (sea) matrix, the crystal structure of PLA could be improved. The possibility of small molecules such as water to penetrate the surface of both crystal structure and the amorphous region would be also increased. They could also have contributed the acceleration of the bio-degradation of the PLA/PHB-blended nonwovens. The relatively high concentrations of the bacterial inoculum and the high inorganic salt concentration in our suspension were the primary reasons for the rapid biodegradation of the PLA/PHBblended nonwovens. The domesticated bacterial community may have increased the degradation of the nonwovens by secreting enzymes that catalyzed the hydrolysis of the ester linkages in the samples. When the macromolecular chains of the PLA/PHB-blended fibers within the nonwovens break down into smaller pieces, bacteria can use the small-molecule substances as nutrient resources during the biodegradation process. ${ }^{32}$ Compared to the samples degrading naturally in soil, the degradation of the polymer samples in the domesticated bacterial suspension was accelerated.

\section{Detection of biodegradation using the domesticated bacterial suspension}

To verify the biodegradability of the PLA/PHB-blended nonwovens in the Gen III soil bacterial suspension, the standard ISO 14852 method was used to determine the amount of evolved $\mathrm{CO}_{2}$. For this experiment, the ratio of $\mathrm{EvCO}_{2}$ to $\mathrm{ThCO}_{2}$ was used to indicate the degree of biodegradation of the PLA/PHBblended nonwovens. The biodegradation of the PLA/PHBblended nonwovens was assessed in the presence of $0.5 \%$ of the Gen III soil bacterial suspension using a ratio of nonwovens to liquid of $1: 40$. As shown in Fig. 3, the percentage of evolved $\mathrm{CO}_{2}$ from the nonwoven samples increased in the presence of the bacterial suspension. The biodegradation of the PLA/PHBblended nonwovens began 3 days after inoculation, and after degrading for 15 days, the amount of $\mathrm{EvCO}_{2}$ reached $566.79 \mathrm{mg}$, indicating a biodegradation yield of $9.93 \%$. In contrast, the percentage of evolved $\mathrm{CO}_{2}$ increased slowly and remained at a low level in the control, where the domesticated bacterial suspension was substituted with sterilized water. The results were similar to those of a previous study ${ }^{24}$ and showed that the renewable PLA/PHB-blended nonwovens could be rapidly degraded and utilized by the domesticated bacterial suspension.

\section{Analysis of OTU abundance and species identification}

The biodegradability of mixed PLA/PHB-blended nonwovens depends on the composition of the degrading microorganisms in the domesticated bacterial suspension. To quantify the
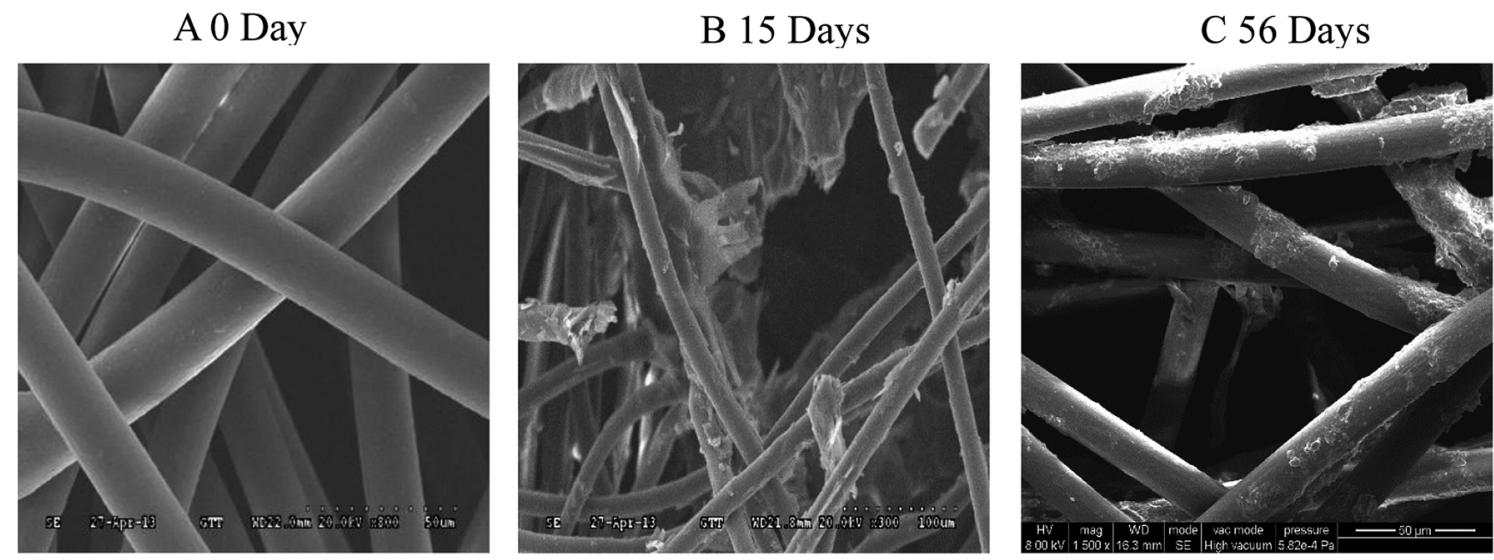

Fig. 2 (A) SEM image of the original PLA/PHB-blended nonwovens $(50 \mu \mathrm{m})$ (B) SEM image of the PLA/PHB-blended nonwovens after 15 days of being immersed in the Gen III soil bacterial suspensions in the lab $(100 \mu \mathrm{m})(C)$ SEM image of the PLA/PHB-blended nonwovens after being buried for 56 days in the soil environment $(50 \mu \mathrm{m})$. 


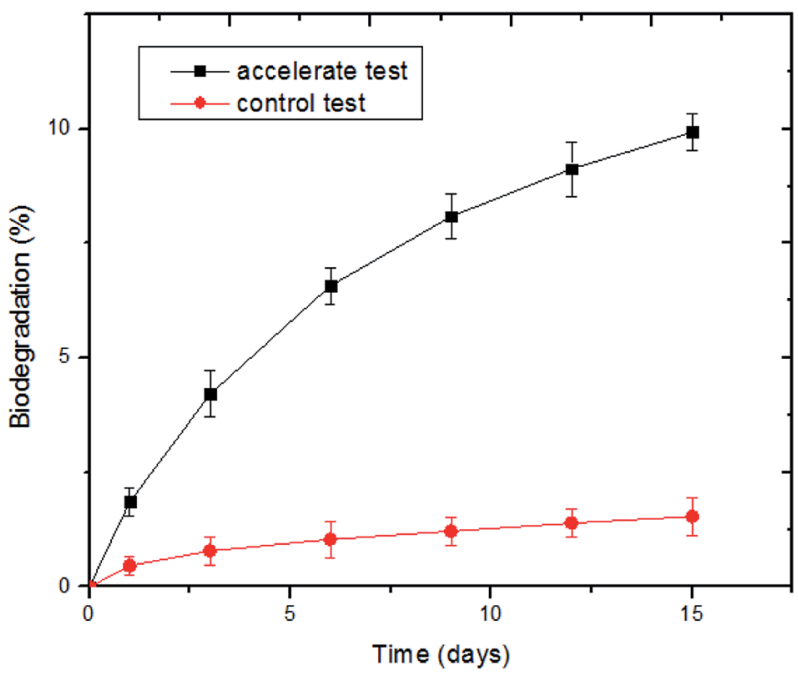

Fig. 3 Percentage of evolved $\mathrm{CO}_{2}$ from the PLA/PHB-blended nonwovens with different degradation time in the presence of the domesticated bacterial suspension.

abundance, diversity and structure of the domesticated bacterial community, a high-throughput pyrosequencing analysis was performed. The amplicon libraries were constructed using primers to amplify the V3 region of the 16S rRNA gene, and high-throughput sequencing was performed on a 454 GS-FLX Titanium protocol to distinguish the bacteria at different taxonomic levels.

After sequencing, $2.5 \mathrm{M}$ raw sequence reads were obtained from the samples, and the corresponding number of bases in the raw sequences was $0.38 \mathrm{~Gb}$. After the connector sequences, low-complexity, and low-quality sequences were removed, $98.1 \%$ of the remaining reads were retained. As shown in Table 2, 9913 sequences were obtained after assembly, and after removing the primers and redundant sequences, 8441 sequences remained. The number of bacterial species was estimated by the number of observed operational taxonomic units (OTUs). In the Gen III samples, 546 OTUs were identified that covered $96.3 \%$ of the remaining sequences. To further study the bacterial community structure, taxonomies of the microbes were analyzed at phylum and genus levels depending on the sequencing data.

\section{Bacterial diversity and community structure analysis}

The distribution of partial bacterial 16S rRNA gene sequences at different levels from the domesticated Gen III sample are shown in Fig. 4. From a total of 8441 sequences obtained from the pyrosequencing analysis, only 2 bacterial phyla were identified, with Proteobacteria and Firmicutes accounting for 80.43 and $19.44 \%$, respectively, representing over $99 \%$ of total classified sequences (Fig. 4A). In the most abundant phylum, Proteobacteria, the unique tag sequences could be classified into 3 orders and 8 genera ( $>1 \%$ of all sequences). At the order level (Fig. $4 \mathrm{~B}$ ), these taxa included Enterobacteriales (70.91\%), Pseudomonadales $(4.73 \%)$, and Aeromonadales (2.86\%). At the genus level (Fig. 4D), the primary genera were Citrobacter (29.4\%), Salmonella (21.00\%), Escherichia (11.70\%), Klebsiella (6.17\%), Pseudomonas (4.73\%), Aeromonas (2.86\%), Enterobacter (1.53\%), and Cronobacter $(1.11 \%)$. In the second most abundant phylum Firmicutes, the unique tag sequences could be classified into 2 orders and 2 genera ( $>1 \%$ of all sequences). At the genus level, these taxa included Lysinibacillus (13.99\%) and Clostridium sensu stricto (3.67\%), which belong to orders Bacillales and Clostridiales, respectively (Fig. 4D).

The above results suggested that due to the abundance of the tag sequences, members of the phyla Proteobacteria (especially those belonging to the genera Citrobacter, Salmonella, Escherichia, and Pseudomonas) and Firmicutes (especially those belonging to the genera Lysinibacillus and Clostridium) may play significant roles in the biodegradation of PLA/PHB-blended nonwovens. Previously, bacterial communities capable of biodegrading mixed PLA and PHB materials were assessed and identified using clone libraries and DNA sequencing to the phylum and class levels. In these studies, the majority of cloned gene sequences belonged to different bacterial phyla, namely, Actinobacteria, Bacteroidetes, Chloroflexi, Firmicutes, Gemmatimonadetes, Planctomycetes, Proteobacteria, and TM7. ${ }^{33,34}$ However, in the present study, only 2 bacterial phyla (Proteobacteria and Firmicutes) were detected. Although the phylum Actinobacteria has often been reported to be capable of biodegrading PLA and other biopolymers, ${ }^{34,35}$ it was not observed in this study. Interestingly, the composition of bacterial phyla obtained from the domesticated cultivation in this study was different from previous results reported from natural soil and composts.

In some cases, the bacterial composition results obtained from cultivation and gene sequencing data were not identical. The microbial diversities identified from the cultivation and gene sequencing methods were different. It is possible that Actinobacteria had a slower growth rate than did strains belonging to the phyla Firmicutes and Proteobacteria. Members of the phyla Firmicutes and Proteobacteria may also rapidly

Table 2 Observed and estimated OTU richness and diversity index for 16S rRNA gene

\begin{tabular}{|c|c|c|c|c|c|c|c|}
\hline \multirow[b]{2}{*}{ Samples } & \multirow[b]{2}{*}{ Seq_num } & \multirow[b]{2}{*}{ Filtered_num } & \multirow[b]{2}{*}{$\begin{array}{l}\text { OTUs coverage } \\
(\%)\end{array}$} & \multirow[b]{2}{*}{ Observed OTUs $^{a}$} & \multicolumn{2}{|c|}{$\begin{array}{l}\text { Estimated OTUs } \\
\text { richness }^{a}\end{array}$} & \multirow[b]{2}{*}{ Shannon $^{a}$} \\
\hline & & & & & ACE & Chao1 & \\
\hline Gen III & 9913 & 8441 & 96.3 & 546 & 1774.8 & 1245.8 & 3.4 \\
\hline
\end{tabular}



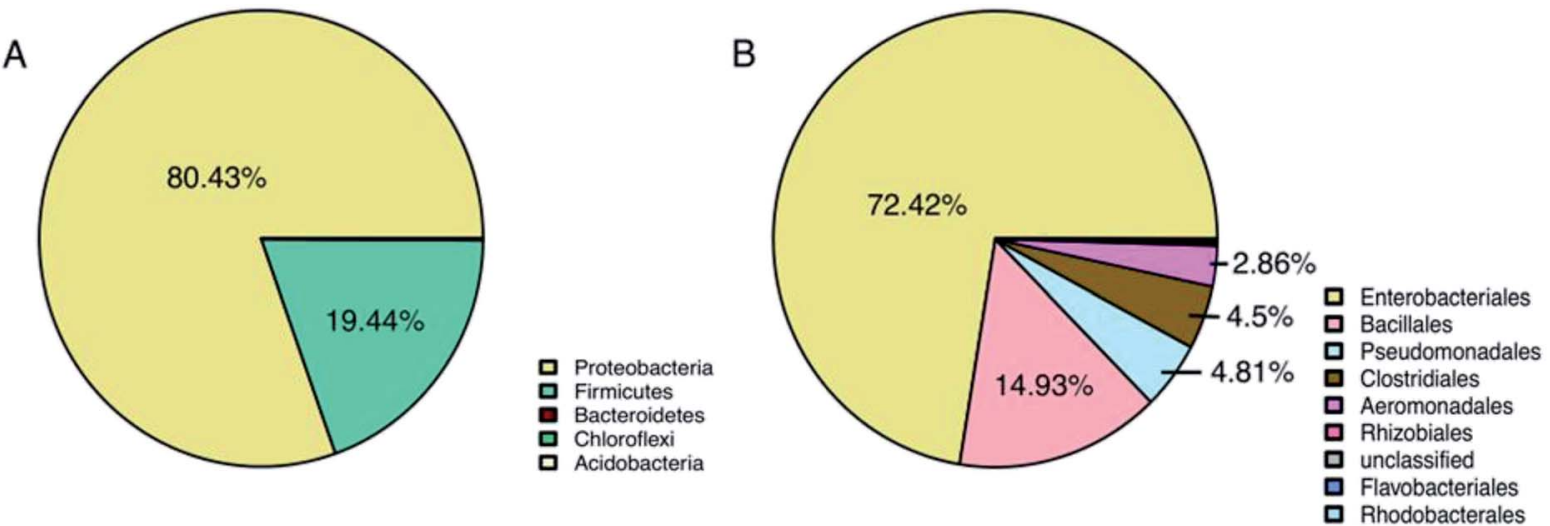

C
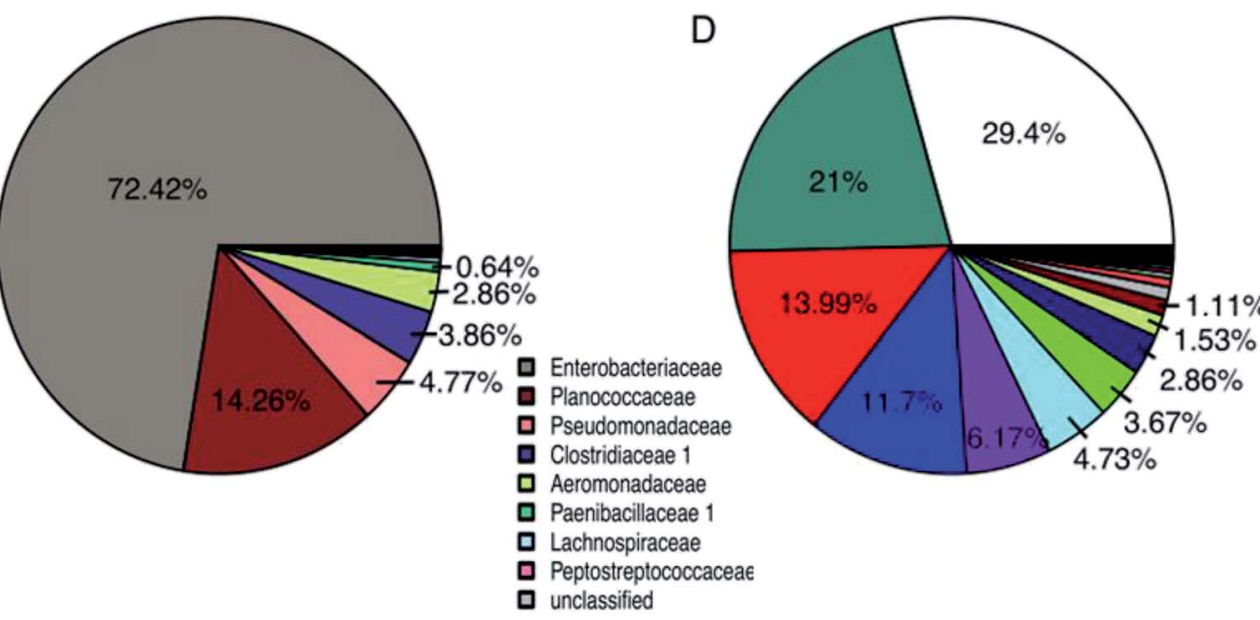

Citrobacter Salmonella Lysinibacillus Escherichia/Shigella - Klebsiella - Pseudomonas 므 Clostridium sensu stricto - Aeromonas Enterobacter - Cronobacter unclassified Paenibacillus 드나idium XIV Kluyvera

Fig. 4 The distribution of partial bacterial 16S rRNA gene sequences at different taxonomic levels from the domesticated bacterial community. (A) Phylum level; (B) order level; (C) family level; and (D) genus level. Proportions were calculated based on the pooled sequences classified with an $80 \%$ confidence threshold.

deplete nutrients from the media that are essential for the growth of Actinobacteria, leading to their eventual failure to grow in the subsequent cultivation. Another possible reason for the absence of Actinobacteria is the sensitivity of members of this phylum to certain media constituents, such as ammonia, amino acids, phosphate, vitamins and salts, or antibiotics produced by other bacteria growing in the cultivation..$^{33,36}$

In the present study, the relative abundance of tag gene sequences in the samples was closely affiliated with members of the genera Citrobacter and Pseudomonas (phylum Proteobacteria) and Lysinibacillus (phylum Firmicutes), indicating that these newly identified bacterial species may play a role in the biodegradation of PLA/PHB-blended nonwovens. Several species belonging to the genus Citrobacter, such as C. amalonaticus, C. koseri, and C. freundii, have been reported to be capable of using polyhydroxy compounds as a sole carbon source. Citrobacter species can also be differentiated by their abilities to convert tryptophan to indole, ferment lactose, and utilize malonate. ${ }^{14}$ Pseudomonas is capable of growing in diesel and jet fuel and can degrade natural rubber, ${ }^{37}$ while Lysinibacillus can degrade xylan..$^{38}$ Although these phylogenetic lineages present in the domesticated cultivation have been reported to play important roles in the degradation of organic matter or polymers, they have not been previously suggested to be associated with the degradation of the PLA/PHB-blended nonwovens.

The results obtained in this study are useful for designing cultivation strategies to accelerate the biodegradation of renewable artificial polymers and may further expand our understanding of the biodegradation mechanisms in domesticated systems. These dominant bacterial strains could be isolated and developed for use in the efficient degradation and recycling of renewable PLA/PHB-blended nonwovens. The isolation of novel strains and the characterization of enzymes produced by these microorganisms will allow for a better understanding of the degradation and cycling of renewable polymer blends in natural environments, such as soil and compost.

\section{Conclusions}

In this study, a method for the rapid degradation of PLA/PHBblended nonwovens using domesticated bacterial strains was demonstrated. The principal components of the bacterial communities in the domesticated cultivation suspensions were identified to reveal the taxa that promoted the biodegradation 
process. Proteobacteria and Firmicutes were the 2 dominant bacterial participants, accounting for 80.43 and $19.44 \%$ of the total classified microbial sequences involved in the biodegradation process, respectively, and may contribute to the high biodegradation of the PLA/PHB-blended nonwovens.

\section{Conflicts of interest}

The authors have no conflicts of interest.

\section{Acknowledgements}

This research was financially supported by the Joint Funds of the National Natural Science Foundation of China (Research project No: U1301231) and the Natural Science Foundation of Guangdong (Research project No: 2013B090600028).

\section{References}

1 T. An, S. Schaeffer, S. Li, S. Fu, J. Pei, H. Li, J. Zhuang, M. Radosevich and J. Wang, Carbon fluxes from plants to soil and dynamics of microbial immobilization under plastic film mulching and fertilizer application using ${ }^{13} \mathrm{C}$ pulse-labeling, Soil Biol. Biochem., 2015, 80, 53-61.

2 W. Sikorska, P. Dacko, M. Sobota, J. Rydz, M. Musio and M. Kowalczuk, Degradation Study of Polymers from Renewable Resources and their Compositions in Industrial Composting Pile, Macromol. Symp., 2010, 272, 132-135.

3 P. Kumar, Role of Plastics on Human Health, Indian J. Pediatr., 2018, 85, 384-389.

4 M. Haward, Plastic pollution of the world's seas and oceans as a contemporary challenge in ocean governance, Nat. Commun., 2018, 9, 667.

5 X. Ping, K. Wang, M. Jia and M. Yang, Biodegradation and mechanical property of polylactic acid/thermoplastic starch blends with poly(ethylene glycol), J. Wuhan Univ. Technol., Mater. Sci. Ed., 2013, 28, 157-162.

6 D. V. Cong, T. Hoang, N. V. Giang, N. T. Ha, T. D. Lam and M. Sumita, A novel enzymatic biodegradable route for PLA/ EVA blends under agricultural soil of Vietnam, Mater. Sci. Eng., C, 2012, 32, 558-563.

7 Y. X. Weng, Y. J. Jin, Q. Y. Meng, L. Wang, M. Zhang and Y. Z. Wang, Biodegradation behavior of poly(butylene adipate-co-terephthalate) (PBAT), poly(lactic acid) (PLA), and their blend under soil conditions, Polym. Test., 2013, 32, 918-926.

8 H. Kang, B. Qiao, R. Wang, Z. Wang, L. Zhang, J. Ma and P. Coates, Employing a novel bioelastomer to toughen polylactide, Polymer, 2013, 54, 2450-2458.

9 S. Zhang, X. Feng, S. Zhu, H. Qian, K. Han, Y. Ma and M. Yu, Novel toughening mechanism for polylactic acid (PLA)/ starch blends with layer-like microstructure via pressureinduced flow (PIF) processing, Mater. Lett., 2013, 98, 238241.

10 V. Nagarajan, A. K. Mohanty and M. Misra, Perspective on Polylactic Acid (PLA) based Sustainable Materials for
Durable Applications: Focus on Toughness and Heat Resistance, ACS Sustainable Chem. Eng., 2016, 4, 2899-2916.

11 M. P. Arrieta, J. López, A. Hernández and E. Rayón, Ternary PLA-PHB-Limonene blends intended for biodegradable food packaging applications, Eur. Polym. J., 2014, 50, 255270.

12 F. Acevedo, P. Villegas, V. Urtuvia, J. Hermosilla, R. Navia and $M$. Seeger, Bacterial polyhydroxybutyrate for electrospun fiber production, Int. J. Biol. Macromol., 2017, 106, 692-697.

13 Y. Tokiwa and T. Suzuki, Hydrolysis of polyesters by lipases, Nature, 1977, 270, 76-78.

14 U. Witt, R. J. Müller and W. D. Deckwer, New biodegradable polyester-copolymers from commodity chemicals with favorable use properties, J. Environ. Polym. Degrad., 1995, 3, 215-223.

15 Y. X. Weng, X. L. Wang and Y. Z. Wang, Biodegradation behavior of PHAs with different chemical structures under controlled composting conditions, Polym. Test., 2011, 30, 372-380.

16 E. Rudnik and D. Briassoulis, Comparative Biodegradation in Soil Behaviour of two Biodegradable Polymers Based on Renewable Resources, J. Polym. Environ., 2011, 19, 18-39.

17 Y. Sadef, T. G. Poulsen and K. Bester, Modeling organic micro pollutant degradation kinetics during sewage sludge composting, Waste Management, 2014, 34, 2007-2013.

18 H. Sun, E. Terhonen, K. Koskinen, L. Paulin, R. Kasanen and F. O. Asiegbu, Bacterial diversity and community structure along different peat soils in boreal forest, Appl. Soil. Ecol., 2014, 74, 37-45.

19 M. K. Gouda, A. E. Swellam and S. H. Omar, Biodegradation of Synthetic Polyesters (BTA and PCL) with Natural Flora in Soil Burial and Pure Cultures under Ambient Temperature, Res. J. Environ. Earth Sci., 2012, 4, 325-333.

20 F. Chen, K. Zhang, H. Zheng, X. Lin, Z. A. Ouyang and T. U. Naimei, Analyzing the Effect of Mixed Decomposition of Conifer and Broadleaf Litters on Soil Microbial Communities by Using PCR-DGGE, Chin. J. Appl. Environ. Biol., 2011, 17, 145-150.

21 H. Zhao, Z. Cui, X. Wang, L. S. Turng and X. Peng, Processing and characterization of solid and microcellular poly(lactic acid)/polyhydroxybutyrate-valerate (PLA/PHBV) blends and PLA/PHBV/Clay nanocomposites, Composites, Part B, 2013, 51, 79-91.

22 C. Luan and C. You, A novel experimental investigation into sintered neck tensile strength of ash at high temperatures, Powder Technol., 2015, 269, 379-384.

23 J. M. Vergès and J. I. Morales, The gigapixel image concept for graphic SEM documentation. Applications in archeological use-wear studies, Micron, 2014, 65, 15-19.

24 W. Guo, J. Tao, C. Yang, Q. Zhao, C. Song and S. Wang, The rapid evaluation of material biodegradability using an improved ISO 14852 method with a microbial community, Polym. Test., 2010, 29, 832-839.

25 G. Muyzer, E. C. de Waal and A. G. Uitterlinden, Profiling of complex microbial populations by denaturing gradient gel electrophoresis analysis of polymerase chain reaction- 
amplified genes coding for $16 \mathrm{~S}$ rRNA, Appl. Environ. Microbiol., 1993, 59, 695-700.

26 B. Dong, J. Yi, L. Dai and X. Dai, Evaluation of Several DNA Extraction Methods for Obtaining Total Community DNA from Anaerobic Digestion Sludge, Procedia Environ. Sci., 2013, 18, 856-863.

27 F. Guo and T. Zhang, Profiling bulking and foaming bacteria in activated sludge by high throughput sequencing, Water Res., 2012, 46, 2772-2782.

28 L. E. S. D. Miera, P. Arroyo, E. D. L. Calabuig, J. Falagán and G. Ansola, High-throughput sequencing of 16S RNA genes of soil bacterial communities from a naturally occurring $\mathrm{CO}_{2}$ gas vent, Int. J. Greenhouse Gas Control, 2014, 29, 176-184.

29 A. M. Bolger, M. Lohse and B. Usadel, Trimmomatic: a flexible trimmer for Illumina sequence data, Bioinformatics, 2014, 30, 2114-2120.

30 R. C. Edgar, UPARSE: highly accurate OTU sequences from microbial amplicon reads, Nat. Methods, 2013, 10, 996.

31 I. Armentano, E. Fortunati, N. Burgos, F. Dominici, F. Luzi, S. Fiori, A. Jiménez, K. Yoon, J. Ahn, S. Kang and J. M. Kenny, Processing and characterization of plasticized PLA/PHB blends for biodegradable multiphase systems, eXPRESS Polym. Lett., 2015, 9, 583-596.
32 M. N. Kim and T. P. Sang, Degradation of poly(L-lactide) by a mesophilic bacterium, J. Appl. Polym. Sci., 2010, 117, 67-74.

33 P. Sangwan and D. Y. Wu, New insights into polylactide biodegradation from molecular ecological techniques, Macromol. Biosci., 2008, 8, 304-315.

34 P. Sangwan, C. Way and D. Y. Wu, New insight into biodegradation of polylactide (PLA)/clay nanocomposites using molecular ecological techniques, Macromol. Biosci., 2009, 9, 677-686.

35 Z. Saadi, A. Rasmont, G. Cesar, H. Bewa and L. Benguigui, Fungal Degradation of Poly(l-lactide) in Soil and in Compost, J. Polym. Environ., 2012, 20, 273-282.

36 W. Amner, C. Edwards and A. J. Mccarthy, Improved medium for recovery and enumeration of the farmer's lung organism, Saccharomonospora viridis, Appl. Environ. Microbiol., 1989, 55, 2669.

37 A. Linos, U. S. A. Keller and R. Reichelt, A Gram-negative bacterium, identified as Pseudomonas aeruginosa AL98, is a potent degrader of natural rubber and synthetic cis-1,4polyisoprene, FEMS Microbiol. Lett., 2000, 182, 155-161.

38 C. S. Lee, Y. T. Jung, S. Park, T. K. Oh and J. H. Yoon, Lysinibacillus xylanilyticus sp. nov., a xylan-degrading bacterium isolated from forest humus, Int. J. Syst. Evol. Microbiol., 2010, 60, 281-286. 